\title{
La Gestión del Proyecto de Bioprótesis Cardiacas del Instituto Nacional de Cardiología-CCADET/ UNAM
}

\author{
Vega-González, R.(I), Juárez Hernández, A. (2), \\ Sánchez-Pérez, E. (3)
}

\begin{abstract}
In this work we present the "Cardiac Innovation Bioprótesis, 2002" technological management project case, developed by the Centro de Ciencias Aplicadas y Desarrollo Tecnológico, CCADET of the Universidad Nacional Autónoma de México, UNAM, for the Instituto Nacional de Cardiología, INC, of the Mexican Health Sector, with funds from the Fondo Sectorial Salud of the Consejo Nacional de Ciencia y Tecnología, CONACYT. The scope and specifications were initially unclear generating complications that become evident through the case development. Littler (2006, pp. II6). We conclude that one of the most important activities of the incubation phase of the project technology management model of the CCADET is the specifications definition.

Keywords: Technology development projects, cardiac valves, Mexican university R\&D centers.

\section{Resumen}

En este trabajo se presenta el caso de la gestión del proyecto "Innovación en Bioprótesis Cardiacas 2002" desarrollado por el Centro de Ciencias Aplicadas y Desarrollo Tecnológico, CCADET de la Universidad Nacional Autónoma de México, UNAM, para el Instituto Nacional de Cardiología, INC, del Sector Salud Mexicano con financiamiento del Fondo Sectorial Salud -CONACYT. A través del desarrollo del caso quedan de manifiesto las complicaciones generadas al no quedar explicitas las especificaciones y los alcanzables de los dispositivos tecnológicos que se requería desarrollar en el proyecto, Littler (2006, pp. I I6). Se concluye que uno de los aspectos más importantes en la gestión tecnológica de proyectos es la definición de especificaciones de la fase de gestación del modelo de Gestión de Proyectos de Desarrollo Tecnológico que se lleva a cabo en el CCADET.
\end{abstract}

Palabras Clave: Proyectos desarrollo tecnológico, bioprótesis cardiacas, Centros universitarios de I\&DT mexicanos.

(I) Centro de Ciencias Aplicadas y Desarrollo Tecnológico, CCADET-UNAM, Coordinación de Vinculación y Gestión Tecnológica; (55)56228602 ext. I I 35 y I I85; Fax 5622-8626; Irvg@servidor.unam.mx

(2) Instituto Nacional de Cardiología, Jefe del Departamento de Biotécnica Aplicada del Instituto Nacional de Cardiología Ignacio Chávez; Tel.

(55) 557329II ext.I3 18, 135I; ajuarez_inc@yahoo.com;

(3) Instituto Nacional de Cardiología, Departamento de Biotécnica Aplicada del Instituto Nacional de Cardiología Ignacio Chávez;

efrit@yahoo.com 


\section{Introducción}

Brasil es un país con 170 millones de habitantes, en el ámbito de la salud tiene alrededor de cuatro mil cirujanos cardiacos y cerca de 90 centros hospitalarios donde se realizan anualmente entre 60 y 70 mil intervenciones de corazón. México, en cambio, cuenta con una población de 110 millones de personas, posee en promedio menos de 100 cardiocirujanos y 15 instituciones en las que se hacen de cinco a seis mil operaciones cardiacas al año; es decir, 90 por ciento menos.

Si bien ambas naciones latinoamericanas son muy similares en materia económica y social, ipor qué Brasil está tan avanzado en ese campo? Sencillo, desde hace varios años dejó de depender exclusivamente de la tecnología extranjera y empezó a fabricar sus propios oxigenadores, tubería para circulación

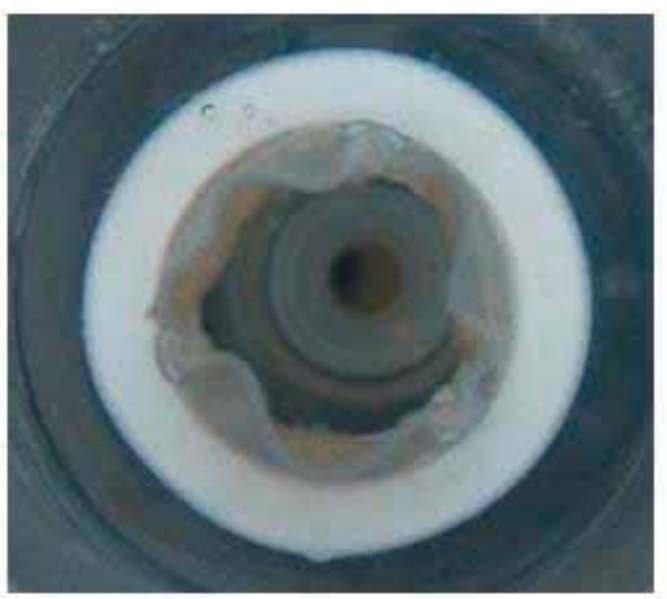

extracorpórea, marcapasos, suturas, y prótesis cardiacas, lo que le ha permitido aumentar el número de cirugías de corazón y reducir los gastos.

Aunque esta dependencia de la Secretaría de Salud es pionera de la cardiología mundial y desde los años 70 ha producido, probado y colocado más de tres mil bioprótesis cardiacas con apoyo de su experimentado cuerpo médico, se reconoce que la demanda de sustitución valvular supera la capacidad del país para atender a los pacientes de escasos recursos. Las bioprótesis actuales son dispositivos biológicos soportados por un anillo rígido de titanio. El primer prototipo también tenía un anillo metálico de stellita que se forraba de duramadre (meninge que cubre al cerebro) de cadáveres humanos y hoy de pericardio (membrana que rodea al corazón) bovino.

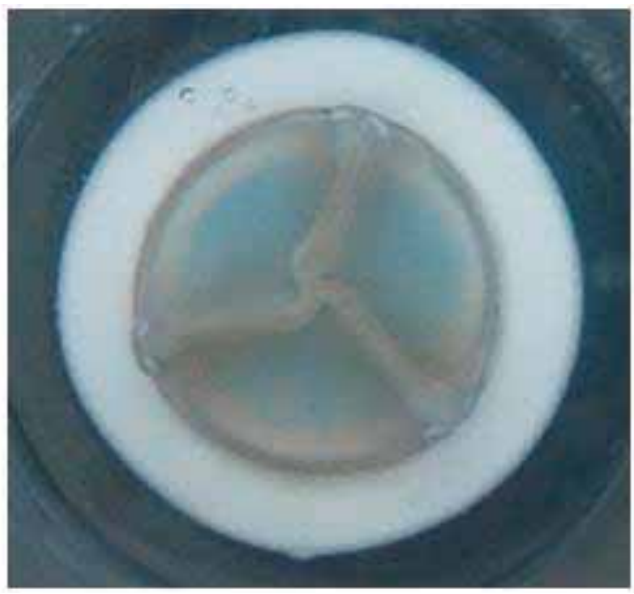

Figura I.Prototipo de bioprótesis cardiaca de pericardio. (Fotografia cortesía del INC)

La mayor parte de la infraestructura para efectuar cirugías a corazón abierto o de mínima invasión proviene de Estados Unidos y Europa, de ahí que cada válvula importada cueste más de tres mil 500 dólares y, aunque corrigen los problemas valvulares tienen otros problemas que las hacen riesgosas. Por ejemplo, la turbulencia que producen las prótesis mecánicas puede producir trombosis y hasta embolias que pueden ser fatales, además necesitan obligadamente el uso de anticoagulantes de por vida, lo cual pone en riesgo de problemas vasculares cerebrales a los pacientes que portan dichas prótesis.

Por otra parte, es un hecho que todas la bioprótesis tienden a calcificarse porque sus tejidos biológicos se preparan con glutaraldehído (un tipo de formol que disminuye el rechazo del xenoinjerto, pero también atrae moléculas de calcio), por esta razón al cabo de una década habrán de reemplazarse por otras. Por ello, el laboratorio de Biotécnica Aplicada del INC, ha desarrollado un sistema para prevenir esta calcificación.

\section{Mejorando las bioprótesis cardiacas}

Después de crear y probar diversos prototipos en busca de una más eficaz, el Departamento de Biotécnica Aplicada inició en 2002 un ambicioso proyecto de innovación enfocado al diseño y manufactura de una nueva generación de bioprótesis cardiacas de pericardio bovino y porcino, dotándolas de un anillo discontinuo fabricado con una aleación de metales base cobalto y un anillo que diera forma y altura correctas a sus postes. Para lograr el apoyo de mayores recursos tecnológicos, se firmó un convenio con la Universidad Nacional Autónoma de México para ser realizado por tres entidades pertenecientes la subsistema de la investigación científica: el Centro de Ciencias Aplicadas y Desarrollo Tecnológico (CCADET), y los institutos de Investigación en Matemáticas Aplicadas y Sistemas (IIMAS) y el de Materiales (IIM). Posteriormente se sometieron propuestas para concurso de recursos a los Fondos Sectoriales en Salud y, a finales del 2002, el Consejo Nacional de Ciencia y Tecnología 
(CONACYT) aprobó un proyecto a tres años el cual concluyó en el 2005.

EI CCADET tomó la responsabilidad de elaborar el equipo automatizado con el que serán fabricados los anillos metálicos, además de un probador para las pruebas hidrodinámicas.

Por su parte, los investigadores del IIMAS construyeron un modelo matemático que predice el comportamiento futuro de las bioprótesis además de un simulador virtual que permitirá indagar sus posibilidades de vida útil. Para ello, emplean una técnica llamada colorimetría, con la cual observan qué regiones del corazón son más vulnerables al estrés, encontrando que por el puro hecho de tener este tipo de anillo, absorbe la mayor parte de la energía de la contracción cardiaca para distribuirla uniformemente en toda la prótesis y nó sólo en la parte biológica de la prótesis, mejorando con ello la sobre vida de la misma en cifras que se calcula que serán hasta de un $30 \%$. Esta parte será tomada en cuenta en el diseño final del anillo $y$, por ende, en su recubrimiento con tejido biológico.

En tanto, al IIM se le asignaron tres subproyectos. El primero fue la obtención de la aleación metálica base cobalto para formar los anillos de las bioprótesis, Posteriormente los técnicos efectuaron todas las pruebas de limpieza, acabado y manufactura de bases valvulares de una sola pieza y de diferentes diámetros usando la aleación desarrollada. El anillo completo sin uniones representa una ventaja en la manufactura de prótesis pues, a diferencia de otras donde se usaba soldadura para unir sus partes, éstas no se fracturarán con los movimientos cardiacos.

El segundo, del cual derivó un primer prototipo, consistió en desarrollar un polímero biocompatible. Por ahora el IIM ya elaboró moldes y realizó las pruebas de inyección, sin embargo aún falta ajustar algunos detalles, como reducir su grosor de 500 a 250 micras, que es la medida requerida para el propósito que se tiene, que es el de completar el anillo de sujeción de la parte biológica.

El tercer proyecto consistió en la caracterización de los tejidos bovinos y porcino, ya que es relevante conocer en qué medida estos materiales resistirán los esfuerzos y las tensiones del corazón.Así, investigadores del IIM fabricaron un probador que evalúa la calidad del pericardio y determina si es viable de usarse en la manufactura de las válvulas o como parche cardiaco. El probador, permitirá saber de qué parte del pericardio podremos obtener el mejor tejido.

Para prolongar hasta por 20 años o más su vida útil y evitar el depósito de sales de calcio, las prótesis finales se someterán a un sistema retardante de la calcificación elaborado por el lab- oratorio de Biotécnica Aplicada del INC, el cual bloquea los sitios de atracción del calcio generados por la presencia de radicales libres del glutaraldehído una vez que cumpla su función. Este proceso llevó cinco años de investigación y ya ha sido probado in vivo e in vitro con resultados favorables.

\section{La Gestión del Proyecto en el CCADET}

En la Figura 2 se presenta Modelo de Gestión de Proyectos de Desarrollo Tecnológico (MGPDT) del CCADET. En el esquema puede verse que en la Fase I o Fase de Gestación del existen varios circuitos de negociación externos. El primero de ellos, CNEI se realiza en la etapa I.I Identificación de Necesidad o demanda (DEM). Esto corresponde a las diversas reuniones IIevadas a cabo entre el personal del Departamento de Bioprótesis Cardiacas del INC y el Coordinador deVinculación del CCADET.

Para dimensionar la magnitud de las necesidades financieras para desarrollar los diferentes sub-proyectos requeridos en el proyecto global de innovación, el Dr. Juárez solicitó que se elaboraran propuestas técnico-económicas.

A partir de este momento se paso a la etapa 1.2 Diagnóstico capacidades Internas (DIA), en la cual se realizaron diversas reuniones internas en el CCADET con el personal académico de los Laboratorios de Electrónica y de Ingeniería de Producto. En estas reuniones quedo definido el equipo técnico de académicos que desarrollaría el proyecto. Estas actividades constituyen el Circuito de Negociación Interno (CNII).

Enseguida se presentó la etapa I.3 Definición de Especificaciones (ESP). Normalmente en esta etapa deben de realizarse nuevas reuniones de intercambio de información entre la parte demandante de la nueva tecnología y quienes la desarrollarán. Esta etapa es muy importante y crucial debido a que siempre existirán ambigüedades. Según Denton (1997), tratar con situaciones ambiguas incrementa la impredicibilidad de los resultados. Por otra parte, Balachandra (1997) encontró a la "definición del problema" como un factor que puede tener influencia positiva o negativa en el éxito en los proyectos de desarrollo e innovación de productos.

Cabe aclarar que los elementos que en ese momento aportó el INC para la presentación de las propuestas de desarrollo fueron la presentación en diapositivas del proyecto por parte del líder del proyecto y el comentario explicito dirigido a los académicos del Laboratorio de Electrónica, de que el probador debería cumplir con la norma mexicana NOM-063-SSAI-I993 sobre bioprótesis cardiacas. 
Posteriormente se realizaron algunas visitas al Laboratorio de Biotécnica Aplicada del INC en la que se mostró a los grupos de trabajo del CCADET un probador de una válvula que había sido donado al INC por un Laboratorio Polaco, y con el cual trabajaban en ese momento para la evaluación de sus válvulas. Por esa razón la estrategia tecnológica que fue adoptada en este proyecto fue la de imitación ya que ante la escasez de recursos se trataba de limitar el riesgo financiero. Schwartz citado por Schewe (1996) encontró desde hace un par de décadas que los imitadores tienen menores costos de desarrollo que los innovadores.

Pero hay otros aspectos positivos en la estrategia de imitación, como son el aprendizaje de otras organizaciones o individuos Schewe (op cit). "Ahora vivimos en la edad del aprendizaje y el crecimiento a partir de otros esfuerzos de cualquier parte del mundo; debemos buscar mejores conceptos, procesos y enfoques para un aprendizaje más efectivo". Kondou, (2003).

Después de que los grupos de trabajo académico analizaron la información, inició la etapa I.4 Propuesta Técnica Económica. Cabe hacer notar que después de analizar la información, los miembros del equipo de académicos del CCADET involucrados en el desarrollo del proyecto manifestaron que aunque no tenían el dominio de los conocimientos y de las habilidades requeridas en el campo de la ingeniería y dispositivos biomédicos especializados en el corazón, tenían el interés de aprender nuevos dominios de conocimiento y habilidades. A esto Danneels (2002) lo llamó competencia de segundo orden. Fue así que después de un plazo no mayor a dos semanas, en Julio de 2002, el CCADET presentó su propuesta para desarrollar un Probador Múltiple de Válvulas Cardiacas (para seis válvulas), según el protocolo entregado por el Laboratorio de Electrónica del Centro. En la segunda partida ofreció el desarrollo de una Máquina automática para la fabricación de anillos.

Los circuitos de negociación tanto internos como externos se repitieron varias veces, por ejemplo, en una reunión posterior se revisó el estado de la gestión del proyecto con la participación del Director del Centro y con el Jefe del Laboratorio de Ingeniería de Producto. En esa reunión el Grupo de técnico de trabajo identificó que en la propuesta técnico-económica no se había incluido el sistema de bombeo para emular el funcionamiento de la válvula y solo se habían considerado la electrónica de potencia y el software de adquisición de datos. De tal manera que se identificó la necesidad de incluir el sistema de bombeo para el probador múltiple, por lo que se invitó a participar al Laboratorio de Micromecánica y Mecatrónica en el proyecto. Este Laboratorio presentó su protocolo de desarrollo por lo que en el mes de septiembre se entregó al INC una nueva propuesta. Nuevamente el tiempo en el cual se presentó la propuesta fue de un par de semanas.

A fines del año 2002 se presentó una buena oportunidad para lograr el financiamiento requerido para el proyecto. Se abrió por primera vez la convocatoria en México para el financiamiento de proyectos dentro del Programa Sectorial Salud. (CONACYT, 2002).

La solicitud de financiamiento se presentó a CONACYT en el mes de noviembre de 2002 incluyendo los requerimientos de todas las propuestas técnico- económicas de las diversas entidades de la UNAM. En abril de 2003, el INC convocó a los responsables de la gestión del proyecto a una reunión general en la que dio a conocer que el CONACYT estaba de acuerdo en asignar los recursos para el proyecto, siempre y cuando todos los participantes redujeran sus requerimientos en un $33 \%$. Se solicitó que en un plazo no mayor a 10 días se respondiera si las diversas entidades de la UNAM participantes estarían de acuerdo en desarrollar el proyecto con ese recorte en el financiamiento.

Inmediatamente se generó una nueva reunión interna en el CCADET (CNIX) entre el Director, con todos los participantes del proyecto y el Jefe del Laboratorio de Ingeniería de Producto y el Coordinador deVinculación. Se acordó que la única forma de aceptar el proyecto sería si se reducían los alcances del mismo.

Los nuevos alcances propuestos para el proyecto fueron:

I. El probador múltiple dejaría de ser de seis válvulas y se convertiría en un probador de una válvula, basado en la NOM-063SSAI-1993-

2. La máquina para la fabricación de anillos dejaría de ser automática y se entregaría en forma semiautomática

3. Se entregarían prototipos funcionales sin incluir ingeniería de producto.

La reducción de alcances y la forma en la que se modificarían los entregables se informó por parte del INC a CONACYT en línea, para estar dentro de los tiempos especificados por ellos mismos en el proceso de evaluación. 


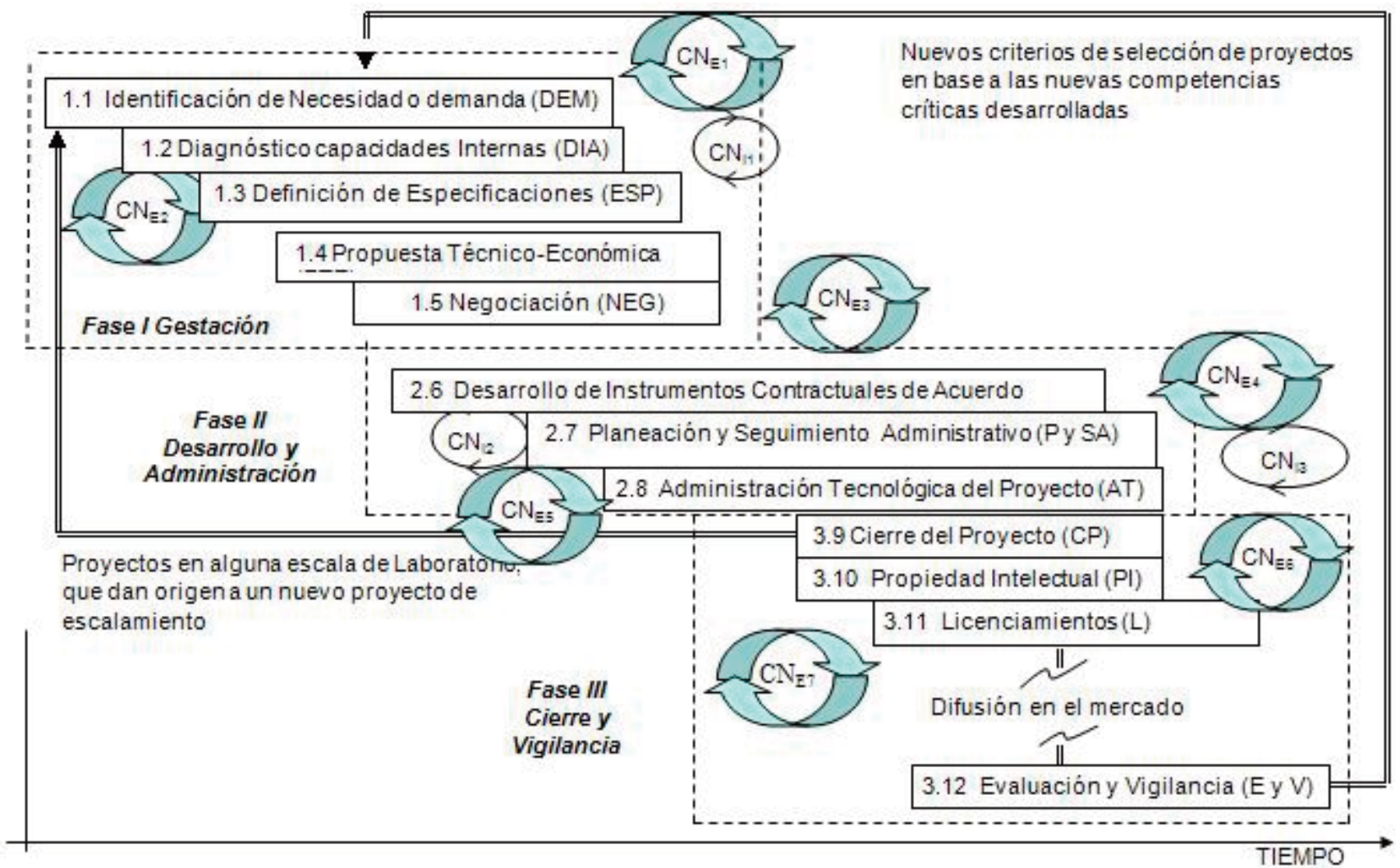

Figura 2 Modelo de Gestión de Proyectos de Desarrollo Tecnológico del CCADET

La Fase 2 del MGPDT, desarrollo y administración del proyecto

En la parte central de la Figura 2 se encuentran las diferentes etapas de la Fase 2 del MGPDT relativas al desarrollo y administración del proyecto. La primera etapa 2.6 consiste en el desarrollo de Instrumentos Contractuales de Acuerdo (CON). Esta parte quedó solventada con los siguientes Convenios:

I. Fondo Sectorial de Salud CONACYT y el INC con la firma del Convenio Sectorial de Salud No. SALUD-2002-COI-8I75, con el objeto de desarrollar el proyecto "Desarrollo e Innovación de Válvulas Cardiacas", con la participación del Instituto de Investigación en Materiales (IIM), del Instituto de Investigación en Matemáticas Aplicadas y Sistemas (IIMAS), y del Centro de Ciencias Aplicadas y Desarrollo Tecnológico (CCADET). La aportación total de CONACYT para el gasto corriente y gasto de inversión del proyecto fue por la cantidad de $\$ 2,999,954.00 \mathrm{MN}$, (dos millones novecientos noventa y nueve mil novecientos cincuenta y cuatro pesos 00/100 MN).

2. Para el mismo efecto señalado en el punto anterior, como se mencionó antes, el INC firmó un Convenio de Colaboración con el Programa Universitario de Investigación en Salud (PUIS), el IIM, el IIMAS y el CCADET, el I0 de abril de 2003, a través del cual se obtuvo un financiamiento concurrente aportado por la UNAM, por la cantidad de $\$ 500,000.00 \mathrm{MN}$ (quinientos mil pesos $00 / 100 \mathrm{MN}$ ). Con lo que el monto total del financiamiento para el proyecto fue de $\$ 3,499,954.00 \mathrm{MN}$ (tres millones cuatrocientos noventa y nueve mil novecientos cincuenta y cuatro pesos 00/100 MN). La mayor parte de este financiamiento concurrente se comprometió para el pago de Becas.

Uno de los primeros problemas que se presentaron fue que CONACYT nunca firmó ningún Convenio con las diferentes entidades de la UNAM involucradas, en el cual quedaran definidas las partidas de gasto corriente y gasto de inversión asignadas para cada caso.

La etapa 2.7 siguiente corresponde a la Planeación y Seguimiento Administrativo (P y SA) de los proyectos desarrollados en el CCADET. Por procedimientos normativos del Fondo Sectorial de Salud, se nombró un administrador único del proyecto en el INC, quien sería responsable de la administración y control de los fondos. Esta situación realmente generó una falta de libertad de acción, lo que complicó mucho el desarrollo del proyecto ya que se generaron tiempos adicionales de espera ante cualquier solicitud de compra de materiales o equipos. 
Al severo problema de administración financiera se agregó la carencia de un Gestor tecnológico quien debería realizar los trabajos involucrados en la etapa 2.8 Administración Tecnológica del Proyecto (AT).

La administración tecnológica del proyecto fue realizada por los Jefes de Laboratorios académicos que participaban en el desarrollo del proyecto y por los responsables por parte del INC quienes convocaban al menos una vez por mes a reuniones de revisión de avances con todo el equipo técnico de desarrollo. Además frecuentemente tenían reuniones de trabajo petit comité con los académicos de los Grupos individuales. De esta forma se presentaron los diferentes informes parciales del proyecto al Fondo sectorial de salud de CONACYT y se llegó al término del proyecto.

\section{Resultados}

Los resultados del CCADET para este proyecto Sectorial CONACYT, obtenidos al mes de Mayo de 2005, fueron un prototipo de probador de bioprótesis funcional, con dos sistemas de impulsión diferentes, con una electroválvula y con un motor de pasos; incluyendo la transmisión mecánica, el software de control y la electrónica necesarios para realizar los ajustes requeridos por las pruebas. También se diseñó una máquina para la fabricación de anillos manual a partir de troqueles que mejoraron la fabricación artesanal. En la Figura 3 se muestran los dispositivos entregados.

El INC inicialmente insistió en que para realizar las pruebas de calidad de sus bioprótesis requería de un probador múltiple que cumpliera con las especificaciones dadas por la norma NOM-063-SSAI-1993.

La norma mencionada indica que para que un substituto de válvula cardiaca pueda certificarse, debe ser sometida a un esquema de evaluaciones en el cual se incluye el accionamiento de ésta en condiciones de flujo que van de 2 a 7 lpm. La norma también solicita que el probador disponga de la instrumentación y de los dispositivos requeridos para realizar las siguientes mediciones: gradiente de presión transvalvular, volumen regurgitante, volumen de fuga y volumen de cierre, volumen minuto y volumen latido, entre otros.

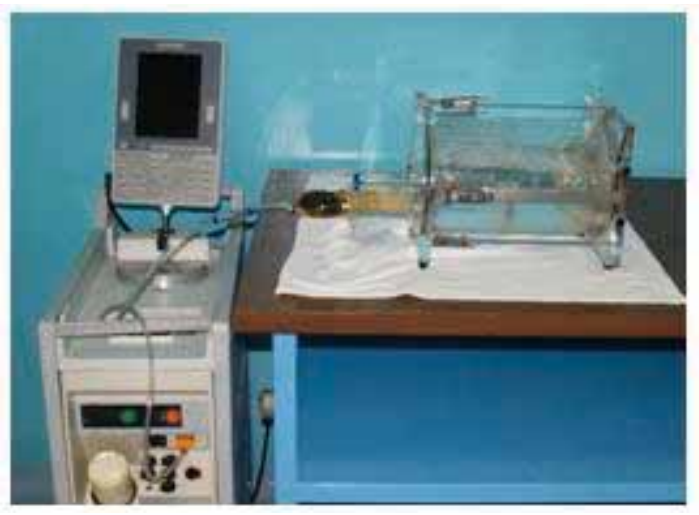

Figura 4 Probador de una válvulas cardiacas usado por el INC

Una de las versiones del probador desarrollado y entregado cuenta con un sistema de impulso basado en un sistema biela manivela para impulsar un pistón al cual se le puede ajustar el flujo por medio del ajuste de su carrera. Cuenta además con un par de sensores de presión los cuales están colocados antes y después de la válvula bajo prueba. En teoría, a través de las mediciones de presión que arrojen estos sensores se podrán determinar las diferentes mediciones que son requeridas para la prueba de válvulas en bradicardia. Pero estas pruebas no se han realizado a la fecha ya que de acuerdo a la percepción de los miembros de los Laboratorios de Electrónica y de Micromecánica y Mecatrónica del CCADET, corresponde al INC realizar la caracterización del equipo debido a que se involucran aspectos de la fisiología del corazón.
El INC también insistió en que además de que se requería el probador para pruebas múltiples, el dispositivo debía permitir la prueba acelerada de las válvulas para agilizar el proceso de estimación de la vida útil y del comportamiento de la bioprótesis en el largo plazo a través del muestreo de los lotes de fabricación.

A primera vista esta diferencia de opinión entre los resultados del CCADET y los requerimientos del INC significaron una falla técnica en el proyecto; sin embargo según Song, et. al., (1997), "ningún plan para el desarrollo de productos puede garantizar el éxito, Cada organización que desarrolla nuevos productos experimentará una buena dosis de fallas parciales o totales". 


\section{La Fase 3 del MGPDT: Cierre y Vigilancia}

El proyecto CONACYT No. SALUD-2002-COI-8I75 inició el 10 de abril de 2003 con una vigencia de 3 años y dos extensiones de un año cada una. El CCADET realizó la entrega oficial de prototipos y manuales de operación el 15 de junio de 2007.

Con esta entrega se dio inició a la etapa 3.9 de Cierre del Proyecto (CP).

Para lograr el acuerdo de cierre con el INC, se realizaron diversas reuniones de trabajo en las cuales se discutieron los siguientes aspectos:

I. La administración y el manejo de un proyecto financiado con fondos del sectorial Salud- CONACYT fueron muy complicados. Financieramente no hubo margen de maniobra económica para el CCADET ya que los rubros financiables no permiten ni el pago de ingresos extraordinarios para los académicos ni el pago de indirectos para la institución. Prácticamente todos los fondos aportados se dedicaron para la compra de materiales requeridos para el desarrollo.

2. Además el presupuesto originalmente asignado para el desarrollo de los tres proyectos del CCADET se quedó demasiado corto ya que fueron utilizados en materiales y algunos equipos requeridos mientras que se utilizó el capital intelectual de entre diez y quince académicos de distintos Laboratorios del Centro así como de alrededor de cinco estudiantes de maestría y de licenciatura.También participaron las áreas de administración, de vinculación e inclusive el propio Director del Centro.

3. Por la razón anterior la aportación intelectual de los académicos que participaron en el desarrollo puede considerarse como una aportación concurrente de la UNAM para el proyecto.

4. El beneficio tangible del proyecto para el CCADET fueron los equipos comprados con los rubros de Gastos de Inversión; sin embargo, los equipos e instrumental que se adquirieron fueron comprados e inventariados por el INC, por lo que como parte de esta etapa del Cierre del Proyecto se requiere la firma de un Convenio de Donación para hacerlos propiedad del CCADET y que puedan ser inventariados por la UNAM.

5. El principal beneficio intangible del proyecto para el CCADET fue el aprendizaje de los diversos grupos académicos de una nueva línea de desarrollo en dispositivos tecnológicos auxiliares para el desarrollo de bioprótesis cardiacas.
6. Una vez desarrollado el proyecto, el Centro obtuvo el "Know How" del desarrollo de los prototipos funcionales de una máquina semiautomática para la fabricación de anillos metálicos, como de un probador de una válvula consistente en un sistema de bombeo y el sistema electrónico de potencia y el software de adquisición de datos.

7. Un problema evidente en la Gestión del proyecto fue la comunicación y el control de minutas del proyecto. Esto evidentemente fue un problema provocado por la falta de un gestor o administrador tecnológico específicamente asignado al proyecto.

8. No existen solicitudes de patente consideradas para la etapa 3. I0 Propiedad Intelectual (PI) de la fase de Cierre y vigilancia, debido a que los desarrollos obtenidos no cumplen con aspectos novedosos o de invención ya que fundamentalmente se trata de la integración de conocimientos diversos que ya son del dominio público. Por la razón anterior por ahora no se ha continuado con la etapa 3.1 I de Licenciamientos (L) ya que los dispositivos desarrollados son para uso del INC y su difusión en el mercado esta restringida.

El cierre del proyecto se pudo concretar cuando tanto el INC como el CCADET reconocieron que uno de los principales logros del proyecto fue el convertirnos en buenos aliados tecnológicos. Ambas partes reconocieron que las propuestas técnico-económicas presentadas al inicio del proyecto se hicieron con la mejor de las intenciones, pero subestimando las problemáticas que podrían darse durante el desarrollo de este tipo específico de dispositivos médicos. Ante la necesidad del INC de obtener los equipos que le ayuden a resolver sus necesidades, la propuesta del CCADET fue la de extender el proyecto hacia una segunda fase con nuevo presupuesto, contemplando un nuevo tiempo de desarrollo, con diferentes entregables específicos y bajo diferentes condiciones de manejo administrativo.

\section{Algunas lecciones aprendidas}

I. El buen resultado de este tipo de proyectos requiere de un buen liderazgo. Desde el inicio del proyecto el liderazgo fue asumido por el Jefe del Departamento de Biotécnica Aplicada del INC, quien mostró una gran capacidad de administración efectiva para alianzas múltiples, según la definición de Rothaermel, et al., (2006).

2. En la gestión de este proyecto quedó muy clara la importancia de la etapa de definición de especificaciones y la necesidad de eliminar la ambigüedad ya que tal y como observamos en el desarrollo del caso. De acuerdo con Hook (2000), existe una relación inversa entre la ambigüedad y la dureza de la realidad. 
3. Debido a los requerimientos de CONACYT en su sistema de Fondos Sectoriales se tuvieron tiempos demasiado cortos para la presentación de propuestas.Además la presentación de las propuestas en línea es un procedimiento ágil pero muy impersonal, ambiguo y los acuerdos no quedan lo suficientemente claros.

4. Por requerimientos normativos de los proyectos Sectoriales de CONACYT, la administración financiera del proyecto se asignó al INC. Esta situación generó muchos problemas debido a que en realidad se tuvo que realizar una doble administración y control, ya que las distintas entidades de cualquier manera cuentan con sus propias áreas Administrativas. Esta situación generó grandes retrasos debido a los tiempos de espera y de actuación requeridos ante cualquier solicitud recompra de materiales y equipos

5. Para mejorar la eficiencia en los tiempos de entrega son necesarios procedimientos logísticos y de administración más ágiles. Aunque ya existía un manual de procedimientos por parte de CONACYT, al trabajar en un proyecto sectorial de primera generación, la práctica administrativa fue muy complicada.

6. De la discusión anterior queda de manifiesto la gran importancia que tiene el contar con un gestor tecnológico para llevar a cabo la administración global del proyecto.

7. Según Littler (2006, pp. I I9), la confianza es un ingrediente esencial de las colaboraciones efectivas. Esta confianza se ha hecho evidente en la relación INC-CCADET/UNAM, ya que ante los resultados y la experiencia alcanzados en la primera fase del proyecto, se han promovido nuevos proyectos con otros grupos académicos. (Zollo, et. al., 2002).

\section{Reflexiones y Conclusión final}

Hacia finales de los años noventa del siglo pasado se reconoció la importancia del ser humano en el proceso de innovación. Drucker (1992), señaló la importancia del conocimiento adquirido por los miembros de las organizaciones, y apuntó que el factor crítico en el proceso de innovación, sería la habilidad de usar el conocimiento científico y tecnológico así como el económico, social y administrativo.

En esa misma época Sengue (1997), inició la corriente del “aprendizaje organizacional”. Según la OCDE (1999), la habilidad de crear, distribuir y explotar el conocimiento y la información será el factor decisivo en el modelo de innovación última generación que se encuentra en proceso y es conocido como el Mileaux innovativo. (Marinova, 2003).
En el caso del proyecto de innovación que hemos presentado, queda de manifiesto que al tratarse de la alianza para desarrollo tecnológico entre dos instituciones del sector público, que no tienen fines de lucro sino de impacto social, el INC y la UNAM, uno de los factores críticos en el proceso han sido, por una parte la decisión y el empuje para iniciar el proyecto por parte del líder del proyecto y la decisión de participar en el proyecto de desarrollo tecnológico, por parte de los diferentes directores, funcionarios y académicos de las entidades de la UNAM involucradas; $y$ por la otra la disponibilidad de recursos financieros. El inicio del proceso fue, sin duda entonces, la decisión de innovar. (Segarra, 1999).

Para evaluar el desempeño de proyectos de desarrollo de nuevos productos Song, et. al., (1997), propusieron criterios como la satisfacción del cliente, la creación de oportunidades, y el crecimiento de largo plazo. Aplicando estos criterios al proyecto CCADET de innovación de válvulas cardiacas los resultados son:

Dado que no hubo satisfacción del cliente en los resultados de la primera fase, para corregir esta situación se propusieron tres proyectos adicionales para la segunda fase de desarrollo del proyecto, lo que se refleja en el criterio de crecimiento de largo plazo.

Creación de oportunidades: este proyecto fue la génesis de una nueva línea de investigación del actual Grupo de Micromecánica y Mecatrónica (M\&M) consistente en el desarrollo de probadores de válvulas cardiacas. También propició la colaboración multidisciplinaria entre los Grupos de Electrónica, M\&M y desarrollo de prototipos así como con el personal del taller mecánico.

Crecimiento de largo plazo: a partir del proyecto inicial consistente en tres sub proyectos, se generaron dos proyectos más que obtuvieron financiamiento del Fondo Sectorial Salud CONACYT 2005 para el desarrollo de un sistema de corte automático de valvas de pericardio bovino con una platina con movimientos bidimensionales y resolución micrométrica y el sistema óptico, de corte con láser.

En conclusión, considerando los diferentes aspectos de evaluación usados internacionalmente, la primera fase del proyecto de innovación de bioprótesis cardiacas INC- CCADET/UNAM fue exitosa ya que abrió las oportunidades de crecimiento en el largo plazo. Estamos en el camino de lograr el objetivo del INC de mejorar sus procesos de fabricación de bioprótesis cardiacas mientras que el CCADET ha hecho su parte para cumplir con uno de los objetivos fundamentales de la UNAM, que es el de coadyuvar en la solución de algunos problemas nacionales. 


\section{Agradecimientos}

Agradecemos el financiamiento para el CCADET recibido del Fondo Sectorial CONACYT SALUD-2002-COI-8I75.

\section{Referencias}

BALACHANDRA, R., (1997), Factors for Success in R\&D Projects and New Product Innovation: A Contextual Framework, IEEE Transactions on Engineering Management. 44(3); pp. 276-287.

CONACYT, (2002?), Programa Sectorial Salud. Disponible en: www.conacyt.mx. (recuperado el 29 de diciembre de 2008)

DANNEELS, E., (2002), The Dynamics of product Innovation and Firm Competences, Strategic Management Journal. 23; pp. I025-I I I I. John Wiley and Sons Ltd.

DENTON, H. G., (1997), Multidisciplinary team-based project work: planning factors, Design Studies, Pp.I55-170. Elsevier Science Ltd.

DRUCKER, P. F, (1992), “The Age of Discontinuity: guidelines to our changing society”, Transaction Publishers, U.S.A.

HOOK, B., (2000), Accomplishment and consumption: ethics for a new millennium, Technology in Society. 22, pp. 477-498. Elsevier Science Ltd.

KONDOU, S., (2003), Striving for Kakushin (continuos innovation) for the 2 Ist century. International Journal of Technology Management. 25(6/7); pp.5 17-530. Inderscience Enterprises Ltd.

LITTLER D; (2006), Alliance enigmas, International Journal of Technology Management, Vol. 33(2/3), pp. I I5-I29.

MARINOVA, D; Phillimore, J., (2003), Models of Innovation, in The Internacional Handbook of Innovation, edited by Larisa V. Shavinina, Pp. 44-53, Elsevier Science Ltd.

Organization for Economic Cooperation and Development, OCDE, (1999) Observer, June.

RIEDEL J., Pawar K., S., (199I), The strategic choice of simultaneous versus sequential engineering for the introduction of new products, International Journal ofTechnology Management, Special Issue on Manufacturing Strategy. 6(3/4), Pp.32I-334.

ROSENBERG N., (1982), Inside the Black Box: the historiography of technical progress, Cambridge University Press, NY, EUA.
ROTHAERMEL, F.T., Deeds, D. L., (2006), Alliance type, alliance experience and alliance management capability in high tech ventures, Journal of Business Venture. 21, pp. 426-460.

ROTHWELL R., (199I), Successful industrial innovation: critical factors for the 1990's, R\&D Management. 22(3), pp. 22I-239.

SCHEWE, G., (1996), Imitation as a strategic option for external acquisition of technology, Journal of Engineering and Technology Management. I 3; pp.55-82. Elsevier Science B.V.

SCHUMPETER, J.,A., (I96I), The theory of economic development, Cambridge Harvard University Press.

SEGARRA, G., (1999), The advanced information technology innovation roadmap, Computers in Industry. 40; pp. I85- 195. Elsevier Science B.V.

SENGUE P. M., (1997), La quinta disciplina: el arte y la práctica de la organización abierta al aprendizaje, Garnica, Barcelona España.

SONG, X., M., Souder,W., E., Dyer, B., (1997), A causal model of the impacts of Skills, Sinergy, and Design Sensitivity on New Products Performance, Journal of Product Innovation Management. 14; pp.88-I0I. Elsevier Science Inc.

VANWINGERDEN, R. G; (200I), Managing Change, International Journal of Technology Management. 2 I (5/6), pp.487-495.

ZOLLO, M., Rever,J..., Singh, H., (2002), Interorganizational routines and performance in strategic alliances. Organization Science. I3, pp. 70I-7I3. 